Kardiologe 2022 $\cdot 16: 178-180$

https://doi.org/10.1007/s12181-021-00510-8

Angenommen: 24. September 2021

Online publiziert: 19. Oktober 2021

๑) Der/die Autor(en) 2021

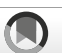

Check for

\section{Hemiparese bei Klappenvegetationen und Panzytopenie}

Liz Kuffer ${ }^{1} \cdot$ Jorge Garcia Borrega ${ }^{2,3} \cdot$ Matthias Kochanek $^{2,3} \cdot$ Felix Kohle $^{4}$.

Clemens Warnke $^{4} \cdot$ Henrik ten Freyhaus ${ }^{1}$

' Klinik III für Innere Medizin, Universität zu Köln, Medizinische Fakultät und Uniklinik Köln, Köln, Deutschland

${ }^{2}$ Klinik I für Innere Medizin, Hämatoonkologie, Centrum für Integrierte Onkologie, Universitätsklinikum Köln, Köln, Deutschland

${ }^{3}$ Klinik I für Innere Medizin, Schwerpunkt Internistische Intensivmedizin, Universitätsklinikum Köln, Köln, Deutschland

${ }^{4}$ Klinik für Neurologie, Universitätsklinikum Köln, Köln, Deutschland

\section{Fallbeschreibung}

Eine 28-jährige Patientin stellte sich aufgrund einer seit dem Vortag bestehenden verwaschenen Sprache und eines hängenden Mundwinkel links vor. Darüber hinaus klagte die Patientin über eine seit mehreren Wochen bestehende Abgeschlagenheit.
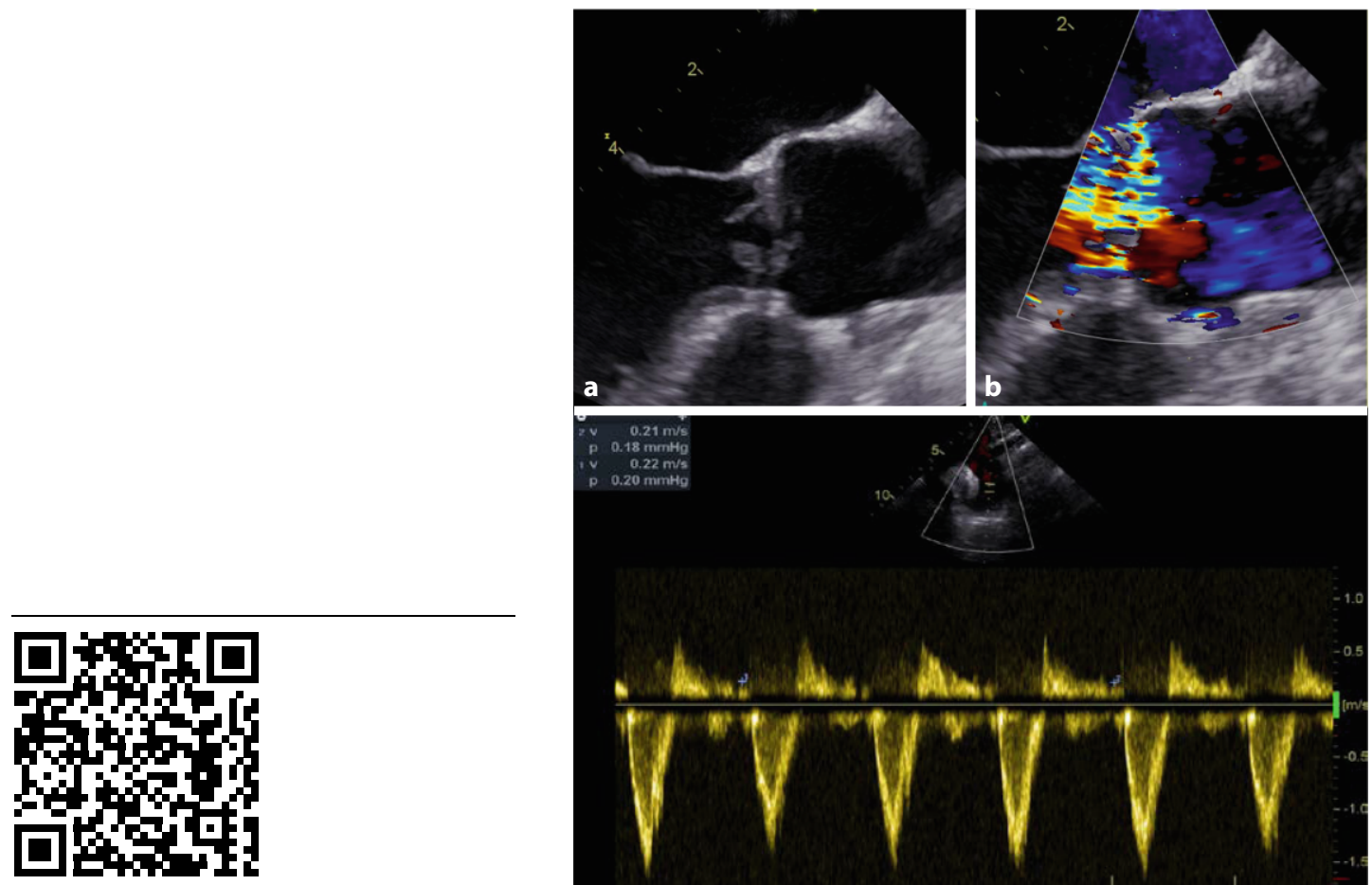

QR-Code scannen \& Beitrag online lesen
Im weiteren Verlauf kam es am Aufnahmetag zu einer linksseitigen Armschwäche.

\section{Klinischer Befund}

Bei Aufnahme wurde in der klinischen Untersuchung eine kardiopulmonal stabile Patientin mit einer zentralen linksseitigen Fazialisparese, einer Dysarthrie und einer armbetonten Hemiparese links gesehen.
Abb. 14 Transösophageale Echokardiographie: a Nachweis mehrerer Vegetationen an der Aoertenklappe mit resultierendem zentralen Koaptationsdefekt, b Nachweis einer hochgradigen Insuffizienz im Farbdoppler. c Transthorakale Echokardiographie mit suprasternaler Anlotung der Aorta descendens mit Nachweis eines relevanten holodiastolischen Refluxes. (Aus [4]; mit freundl. Genehmigung von Coxford University Press 2020. All rights reserved) 


\section{Diagnostik}

\section{Perfusions-CT-Schädel}

Infarktdemarkation im vorderen Mediastromgebiet rechts. Proximaler M2-Abbruch. Kein Nachweis einer Penumbra in der Perfusion.

\section{Aufnahmelabor}

Im Aufnahmelabor zeigte sich eine Thrombozytopenie $\left(51 \times 10^{9} / \mathrm{l}\right)$, sowie eine milde Anämie und eine Leukozytopenie (jeweils $10 \mathrm{~g} / \mathrm{dl}$ und $\left.4,0 \times 10^{9} / \mathrm{l}\right)$. Das CRP war leicht erhöht $(35,3 \mathrm{mg} / \mathrm{l})$.

\section{Transösophageale Echo-} kardiographie

Trikuspide Aortenklappe mit deutlich aufgetriebenen und verplumpten Taschen- rändern sowie kleineren flottierenden Vegetationen an allen Taschen (maximale Länge ca. $7 \mathrm{~mm}$ ) mit resultierendem zentralen Koaptationsdefekt. Hochgradige Insuffizienz, holodiastolischer Reflux in der Aorta descendens (- Abb. 1). Linker Ventrikel normal dimensioniert mit guter systolischer Funktion. Keine Thromben im LA oder LAA.

\section{Mikrobiologische Diagnostik}

Bei allzeit afebriler Patientin blieben alle entnommenen Blutkulturen ohne Erregernachweis. Serologisch konnte kein H.a. das Vorliegen einer Infektion mit Bartonella spp., Brucella spp., Coxiella burnetii, Legionella pneumophila und Mycoplasma pneumonia gesehen werden.

\section{Wie lautet Ihre Diagnose?}

\section{Therapie und Verlauf}

Aufgrund der lang bestehenden Symptomatik und des fehlenden Nachweises einer Penumbra konnten bei gleichzeitig vorliegender Thrombozytopenie weder eine Lyse noch eine mechanische Thrombek-

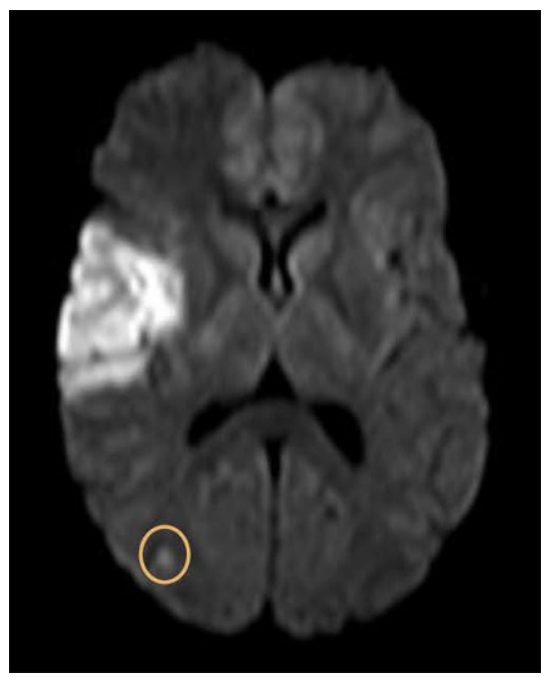

Abb. $2 \Delta$ Axiale diffusionsgewichtete cMRTBildgebung mit Nachweis eines rechtshemispherischen Infarkts des M2-Stromgebiets und einem kleineren parietookzipitalen Infarkt. (Aus [4]; mit freundl.Genehmigung von $\odot$ Oxford University Press 2020. All rights reserved) tomie durchgeführt werden. Unmittelbar nach Stellen der Verdachtsdiagnose APL erfolgte die notfallmäßige Einleitung einer Therapie mit All-trans-Retinoinsäure, Arsentrioxid und Prednisolon [5]. Darüber hinaus wurde eine therapeutische Antikoagulation durchgeführt.

Eine Verlaufs-cMRT (kraniale Magnetresonanztomographie) zeigte ein demarkiertes Infarktareal des rechtshemispherischen M2-Territorials sowie eine kleinere parietookzipitale Infarktdemarkation (• Abb. 2) ohne Einblutung.

Aufgrund der fortbestehenden Emboliegefahr und der hochgradigen Aortenklappeninsuffizienz wurde ein operativer Klappenersatz interdisziplinär diskutiert. Doch aufgrund des erhöhten Blutungsrisikos im Rahmen der Thrombozytopenie wurde bei hämodynamisch stabiler Patientin für die Fortführung eines konservativen Procedere entschieden.

Im Weiteren kam es unter der bestehenden Chemotherapie und Antikoagulation erfreulicherweise zu einer vollständigen Regredienz der Aortenklappenvegetationen mit einer funktional kompetenten Aortenklappe (-Abb. 3). Es traten keine weiteren klinisch apparente Thrombembolien auf.

\section{Weitere Labordiagnostik}

Normalbefunde für ANA, Anti-dsDNA, AntiSm, C3- und C4-Komplementfaktoren, Anti-ß2-Glykoprotein-I-Antikörper, Anti-Cardiolipin-Antikörper, aPTT.

Mikroskopisches Differenzialblutbild: vermehrter Nachweis stabkerniger neutrophiler Granulozyten (26\%) im Sinne einer Linksverschiebung bei Thrombound Lymphozytopenie.

\section{Knochenmarkpunktion und Molekulargenetik}

Infiltration von $70 \%$ des Knochenmarks durch atypische Promyelozyten, Nachweis von Faggot-Zellen. Im weiteren Verlauf konnte mittels PCR eine hohe Expression eines $P M L / R A R A$-Fusionsgens nachgewiesen werden.

\section{Definition}

Die NBTE, oft auch als marantische Endokarditis oder Libman-Sacks-Endokarditis bezeichnet, stellt eine Krankheitsentität dar, bei der es zu sterilen Vegetationen an den Herzklappen kommt. Von diesen aus Fibrin und aggregierten Thrombozyten bestehenden Vegetationen geht, anders als bei der infektiösen Endokarditis, keine Destruktion des Klappenapparates aus, allerdings liegt jedoch ein erhöhtes thrombembolisches Risiko vor [6].

Die NBTE tritt vor allem - wie bei der hier vorgestellten Patientin - im Rahmen von malignen Erkrankungen und bei Patienten mit einem systemischem Lupus erythematodes oder einem Antiphospholipidsyndrom auf. Die Diagnosestellung ist oftmals nicht trivial, wobei hier insbesondere die Abgrenzung zur kulturnegativen infektiösen Endokarditis wichtig ist. Die häufigsten Ursachen für letztere stellen eine bereits bestehende antibiotische Therapie zum Zeitpunkt der Blutkulturentnahme und das Vorhandensein von nicht bzw. schwer anzüchtbaren Bakterien dar [2, 3].

Die Behandlung besteht grundsätzlich aus der Therapie der Grunderkrankung und einer Antikoagulation, wobei gera- 


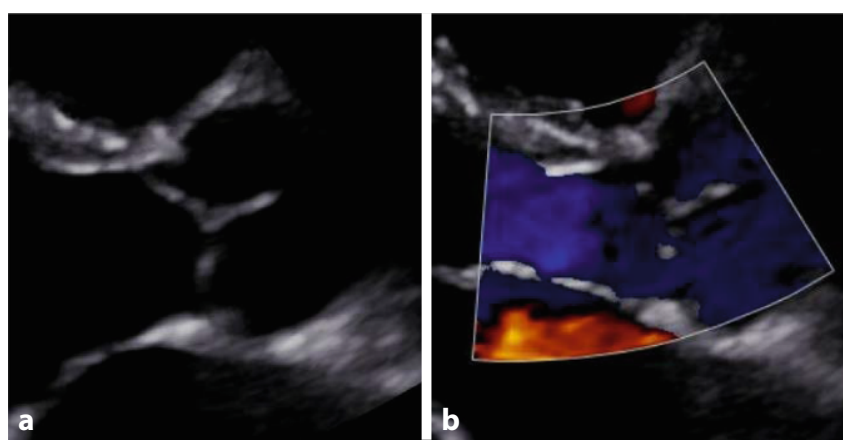

Abb. $3 \Delta$ Transthorakale Echokardiographie mit a vollständiger Regredienz der Vegetationen an der Aortenklappe und normaler Koaptation der Taschen sowie b ohne Nachweis einer Insuffizienz im Farbdoppler. (Aus [4]; mit freundl. Genehmigung von @Oxford University Press 2020. All rights reserved)

de letztere im klinischen Alttag aufgrund der Grund- und Nebenerkrankungen eine Herausforderung darstellen kann. So ist die bei der vorgestellten Patientin bestehende APL klassischerweise mit Blutungskomplikationen vergesellschaftet. Die Frage des optimalen Zeitpunkts für den Beginn einer oralen Antikoagulation nach stattgehabtem Schlaganfall im Hinblick auf sekundäre Hämorrhagien ist durch die aktuelle Datenlage noch nicht endgültig geklärt $[1,7]$. Darüber hinaus liegt zum weiteren Vorgehen bei gleichzeitig vorliegenden hochgradigen Klappenvitien für den Sonderfall der NBTE keine robuste Evidenz vor. In der Literatur ist lediglich eine Reihe an Einzelfallberichten mit überwiegend operativen Therapien beschrieben. Der erfreuliche Verlauf mit kompletter Regredienz der Vegetationen und Erholung der Klappenfunktion bei der vorgestellten $\mathrm{Pa}$ tientin zeigt jedoch, dass ein konservatives Vorgehen eine durchaus zu diskutierende Therapieoption darstellt.

\section{/) Diagnose: nichtbakterielle thrombotische Endokarditis (NBTE) bei akuter Promyelozytenleukämie (APL) mit thromboembolischem Schlaganfall}

Dabei ist aufgrund der Komplexität des Krankheitsbildes sowie der aus verschiedenen Fachrichtungen $z u$ beachtenden Aspekten sowohl für die Diagnosestellung als auch für die optimale Therapie von Patienten mit NBTE eine engmaschige interdisziplinäre Zusammenarbeit entscheidend.

\section{Fazit für die Praxis}

- Die NBTE (nichtbakterielle thrombotische Endokarditis) stellt eine seltene Ursache für Schlaganfälle und eine Differenzialdiagnose von Klappenvegetationen dar.

- Eine konservative Therapie anstelle eines Klappenersatzes sollte auch bei hochgradigen Klappenvitien mit stattgehabten Embolien als Therapieoption diskutiert werden.

- Ein interdisziplinäres Vorgehen mit Onkologen, Neurologen, Rheumatologen, Nephrologen und ggf. Mikrobiologen ist zur Diagnosesicherung und Therapie unabdingbar.

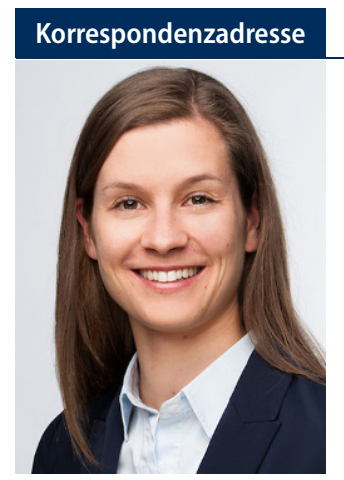

\section{Dr. med. Liz Kuffer}

Klinik III für Innere Medizin, Universität zu Köln, Medizinische Fakultät und Uniklinik Köln Kerpener Straße 62, 50937 Köln, Deutschland liz.kuffer@uk-koeln.de

Funding. Open Access funding enabled and organized by Projekt DEAL.

\section{Einhaltung ethischer Richtlinien}

Interessenkonflikt. L. Kuffer, J. Garcia Borrega, M. Kochanek, F. Kohle, C. Warnke und H. ten Freyhaus geben an, dass kein Interessenkonflikt besteht.
Für diesen Beitrag wurden von den Autoren keine Studien an Menschen oder Tieren durchgeführt. Für die aufgeführten Studien gelten die jeweils dort angegebenen ethischen Richtlinien. Für Bildmaterial oder anderweitige Angaben innerhalb des Manuskripts, über die Patienten zu identifizieren sind, liegt von ihnen und/oder ihren gesetzlichen Vertretern eine schriftliche Einwilligung vor.

Open Access. Dieser Artikel wird unter der Creative Commons Namensnennung 4.0 International Lizenz veröffentlicht, welche die Nutzung, Vervielfältigung, Bearbeitung, Verbreitung und Wiedergabe in jeglichem Medium und Format erlaubt, sofern Sie den/die ursprünglichen Autor(en) und die Quelle ordnungsgemäß nennen, einen Link zur Creative Commons Lizenz beifügen und angeben, ob Änderungen vorgenommen wurden.

Die in diesem Artikel enthaltenen Bilder und sonstiges Drittmaterial unterliegen ebenfalls der genannten Creative Commons Lizenz, sofern sich aus der Abbildungslegende nichts anderes ergibt. Sofern das betreffende Material nicht unter der genannten Creative Commons Lizenz steht und die betreffende Handlung nicht nach gesetzlichen Vorschriften erlaubt ist, ist für die oben aufgeführten Weiterverwendungen des Materials die Einwilligung des jeweiligen Rechteinhabers einzuholen.

Weitere Details zur Lizenz entnehmen Sie bitte der Lizenzinformation auf http://creativecommons.org/ licenses/by/4.0/deed.de.

\section{Literatur}

1. Ahmed N, Steiner T, Caso V, Wahlgren N, ESOKSU SP (2017) Recommendations from the ESOKarolinska Stroke Update Conference, Stockholm 13-15 November 2016. Eur Stroke J 2:95-102

2. Fournier PE et al (2010) Comprehensive diagnostic strategy for blood culture-negative endocarditis: a prospective study of 819 new cases. Clin Infect Dis 51:131-140

3. Habib G et al (2015) 2015 ESC Guidelines for the management of infective endocarditis: The Task Force for the Management of Infective Endocarditis of the European Society of Cardiology (ESC). Endorsed by: European Association for Cardio-Thoracic Surgery (EACTS), the European Association of Nuclear Medicine (EANM). Eur Heart J 36:3075-3128

4. Kuffer L et al (2020) P630 Recovery of nonbacterial thrombotic endocarditis and severe aortic regurgitation in a young patient with acute promyelocytic leukemia. Eur Heart J Cardiovasc Imaging. https://doi.org/10.1093/ehjci/jez319. 314 (EuroEcho 2019 Abstract Supplement)

5. Lo-Coco F et al (2013) Retinoic acid and arsenic trioxide for acute promyelocytic leukemia. N Engl J Med 369:111-121

6. Reisner SA, Brenner B, Haim N, Edoute Y, Markiewicz W (2000) Echocardiography in nonbacterial thrombotic endocarditis: from autopsy to clinical entity. J Am Soc Echocardiogr 13:876-881

7. Seiffge DJ et al (2019) Timing of anticoagulation after recent ischaemic stroke in patients with atrial fibrillation. Lancet Neurol 18:117-126 